Rev. High Pressure Sci. Technol., Vol. 7 (1998) 547 549

\title{
Pressure Effect on the Curie Temperature of $\mathrm{Ni}_{2} \mathrm{In}-\mathrm{Type}\left(\mathrm{Mn}_{1-z} \mathrm{Fe}_{z}\right)_{7} \mathrm{Sn}_{4}$
}

\author{
S.Anzai, S.Fujii, S.Ohta*, H.Yoshida+, T. Kaneko+, R.Sugi, \\ T.Yoshida, M.Matoba, and T.Wada \\ Faculty of Science and Technology, Keio University, Hiyoshi, Yokohama-city, 223, Japan \\ *Faculty of Engineering, Yamagata University, Jonan, Yonezawa-city, 992, Japan \\ +Institute for Materials Research, Tohoku University, Katahira, Sendai-city, 980-77, Japan
}

\begin{abstract}
The variations of the Curie temperature $T_{\mathrm{c}}$ and ferrimagnetic moment $n_{\mathrm{f}}$ in $\left(\mathrm{Mn}_{1-z} \mathrm{Fe}_{z}\right)_{7} \mathrm{Sn}_{4}(0 \leqq z \leqq 0.35)$ have been investigated as functions of $z$ and hydrostatic pressure $P$ up to the maximum $12.5 \mathrm{kbar}$. Below $z \sim 0.25, T_{\mathrm{c}}$ decreases with increasing $z$, but it increases above $\mathrm{z} \sim 0.25$. The pressure derivative of $T_{\mathrm{c}}, \mathrm{d} T_{\mathrm{c}} / \mathrm{d} P$ decreases and $n_{\mathrm{f}}$ increases with increasing $z$ below $z \sim 0.1$ above which these values are nearly constant. These features are compared with the results of $\left(\mathrm{Mn}_{1-u} \mathrm{Co}_{u}\right)_{7} \mathrm{Sn}_{4}$ and $\mathrm{Mn}_{7}\left(\mathrm{Sn}_{1-x} \mathrm{Ge}_{x}\right)_{4}$ from the electronic and magnetic points of view.

[pressure effect, Curie temperature, ferrimagnetism, $\left(\mathrm{Mn}_{1-2} \mathrm{Fe}_{2}\right) \mathrm{Sn}_{4}$, itinerant electron magnet]
\end{abstract}

\section{Introduction}

The $\mathrm{Ni}_{2}$ In-type $\mathrm{Mn}_{7} \mathrm{Sn}_{4}$ is ferrimagnetic below the Curie temperature $T_{\mathrm{c}}=230 \mathrm{~K}[1]$. According to the neutron diffraction study[2], the magnetic moments at $\mathrm{MnI}$ sites are ferromagnetically aligned in the basal plane while those at MnII sites are antiferromagnetically coupled with those at MnI sites. Here, in $\mathrm{Mn}_{7} \mathrm{Sn}_{4}$, there are two different types of $\mathrm{Mn}$ sites; $\mathrm{MnI}$ and MnII, as shown in the inset of Fig.1. The ferrimagnetic moment $n_{\mathrm{f}}$ comes from the difference in Mn moments, $m(\mathrm{Mn})$,
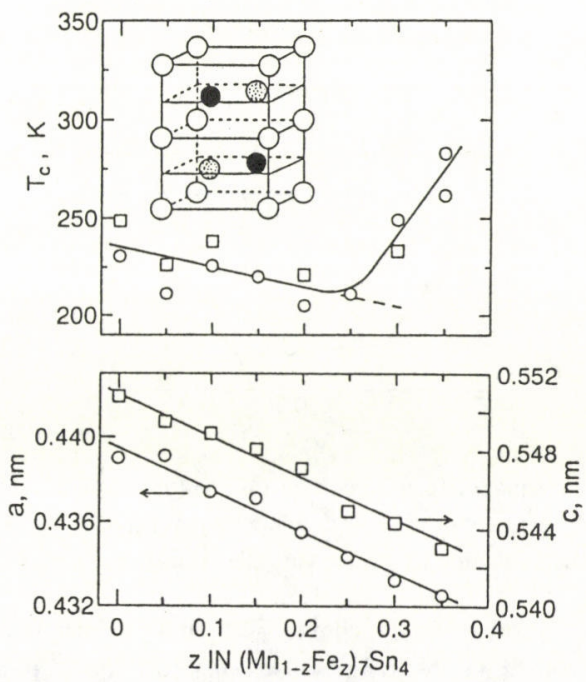

Fig.1. Curie temperature $T_{\mathrm{c}}$ (upper panel); Ofrom the $\sigma-T$ curve; $\square$ from the $\mu-T$ curve and lattice parameters $a$ and $c$ (lower panel) as a function of $z$ in $\left(\mathrm{Mn}_{1-2} \mathrm{Fe}_{2}\right)_{7} \mathrm{Sn}_{4}$. Inset shows the $\mathrm{Ni}_{2}$ In-type structure. Here, open, closed and dotted circles represent for $\mathrm{MnI}, \mathrm{MnII}$ and $\mathrm{Sn}$ atoms, respectively.

between $\mathrm{MnI}$ and $\mathrm{MnII}$ sites: $m(\mathrm{MnI})=0.8 \mu_{\mathrm{B}}$ and $m(\mathrm{MnII})=3.8$ $\mu_{\mathrm{B}}$. An X-ray photoemmision investigation on the $\mathrm{Ni}_{2}$ In-type ferrimagnetic materials $\mathrm{Mn}_{7-y} \mathrm{Sn}_{4}$ shows that $3 d$ electrons of $\mathrm{Mn}$ contribute to the Fermi edge[3]. Pressure contraction raises $T_{\mathrm{c}}$ in $\mathrm{Mn}_{7} \mathrm{Sn}_{4}[4]$. On the other hand, the $\mathrm{Ni}_{2} \mathrm{In}$-type $\mathrm{Fe}_{1+\delta} \mathrm{Sn}(\delta>0)$ is ferromagnetic below $T_{\mathrm{c}} \sim 700 \mathrm{~K}$, in which the saturation magnetization per $\mathrm{Fe}$ atom is $1.8 \mu_{\mathrm{B}}[5]$. From the Mössbauer spectra, the values of $m(\mathrm{FeI})=1.9 \mu_{\mathrm{B}}$ and $m(\mathrm{FeII})=1.8 \mu_{\mathrm{B}}$ have been estimated[6]. In order to study the stability of magnetic states in metallic solids, we report the $z$ and $P$ (pressure) dependences of $T_{\mathrm{c}}$, together with the $z$ dependence of $n_{\mathrm{f}}$, in $\left(\mathrm{Mn}_{1-2} \mathrm{Fe}_{2}\right)_{7} \mathrm{Sn}_{4}$, and compare them with the cases of $\left(\mathrm{Mn}_{1}\right.$. $\left.{ }_{u} \mathrm{Co}_{u}\right)_{7} \mathrm{Sn}_{4}[7,8]$ and $\mathrm{Mn}_{7}\left(\mathrm{Sn}_{1-x} \mathrm{Ge}_{x}\right)_{4}[9,10]$.

\section{Experimental methods}

The methods of sample preparations and magnetic measurements were described in the previous paper[9]. The permeability $(\mu)$ of the samples was measured under hydrostatic $\operatorname{pressure}(P)$ up to the maximum $12.5 \mathrm{kbar}$. Here, the generation of pressure was performed with a piston-cylinder type apparatus. Fluorinart(\#75) was used as a high-pressure medium. The powder X-ray diffraction pattern for the samples obtained can be explained in terms of the $\mathrm{Ni}_{2}$ In-type crystal structure and no foreign peak was detected.

\section{Results}

As seen in the upper panel of Figure 1, $T_{c}$ decreases with increasing $z$ up to a critical concentration $z_{c}=0.25$ above which it turns to increase up to $z=0.35$. Figures 2 and 3 show $\mu$ as a function of temperature $(T)$ at typical pressures for the $z=0.05$ and 0.1 samples and the $z=0.2$ and 0.3 ones, respectively. As $P$ increases, the sharp peak shifts to the higher temperature range. Here, $T_{\mathrm{c}}$ is assigned from the intersection of two lines drawn in the $\mu-T$ curve. Figure 4 shows $T_{\mathrm{c}}$ as a function of $P$ for various $z$ samples, in which $T_{\mathrm{c}}$ increases with increasing $P$. Below $P \sim$ $5 \mathrm{kbar}$, non reproducible features of $T_{\mathrm{c}}$ have been reported on the data for $\mathrm{Mn}_{7}\left(\mathrm{Sn}_{1-x} \mathrm{Ge}_{x}\right)_{4}[10]$ and $\left(\mathrm{Mn}_{1-u} \mathrm{Co}_{u}\right)_{7} \mathrm{Sn}_{4}[8]$. Hence, the data of $T_{\mathrm{c}}(P>5 \mathrm{kbar})$ are discussed hereafter. As seen in the lower panel of Figure 5 , the pressure derivative of $T_{\mathrm{c}}, \mathrm{d} T_{\mathrm{c}} / \mathrm{d} P$, steeply decreases with increasing $z$ in the range $0 \leqq z \leqq 0.1$, while it 
slowly changes in $0.1<z \leqq 0.3$. The least squares fitting to the $\mathrm{d} T_{\mathrm{c}} / \mathrm{d} P$ against $z$ in the range $0 \leqq z \leqq 0.1$ gives the value of $\mathrm{d}\left(\mathrm{d} T_{\mathrm{c}} / \mathrm{d} P\right) / \mathrm{d} z=-9.5 \times 10^{-2}(\mathrm{~K} / \mathrm{kbar}) / \mathrm{at} \%$. This value is closer to that $\left(\mathrm{d}\left(\mathrm{d} T_{\mathrm{c}} / \mathrm{d} P\right) / \mathrm{d} u=-6.0 \times 10^{-2}(\mathrm{~K} / \mathrm{kbar}) / \mathrm{at} \%\right)[8]$ for $\left(\mathrm{Mn}_{1-\mathrm{u}} \mathrm{Co}_{\mathrm{u}}\right) / 7$ $\mathrm{Sn}_{4}$ but considerably larger than that $\left(\mathrm{d}\left(\mathrm{d} T_{\mathrm{c}} / \mathrm{d} P\right) / \mathrm{d} x=-1.7 \times 10^{-2}\right.$ $(\mathrm{K} / \mathrm{kbar}) / \mathrm{at} \%)[10]$ for $\mathrm{Mn}_{7}\left(\mathrm{Sn}_{1-x} \mathrm{Ge}_{x}\right)_{4}$. Figure 6 shows the magnetization $\sigma$ at $6 \mathrm{~K}$ as a function of magnetic field $(H)$ up to 6T. As $H$ increases, $\sigma$ steeply increases and then saturates in the higher $H$ range. These data are analyzed on the basis of the

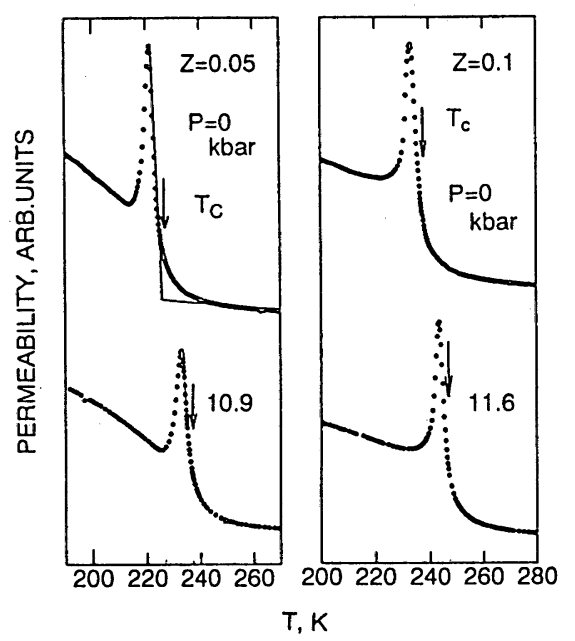

Fig.2. Permeability as a function of temperature $T$ for the $z=0.05$ and 0.1 samples in $\left(\mathrm{Mn}_{1-2} \mathrm{Fe}_{2}\right)_{7} \mathrm{Sn}_{4}$ at typical pressures $P$.

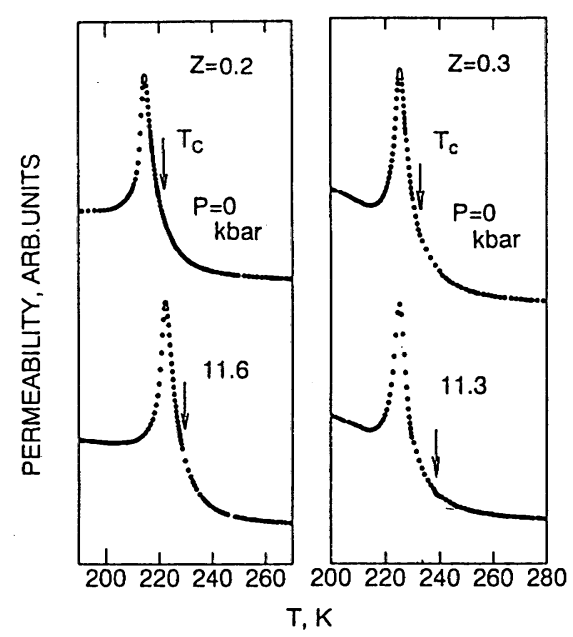

Fig.3. Permeability as a function of temperature $T$ for the $z=0.2$ and 0.3 samples in $\left(\mathrm{Mn}_{1-2} \mathrm{Fe}_{2}\right)_{7} \mathrm{Sn}_{4}$ at typical pressures $P$.
Arrott plots which is written as $\sigma^{2}=\alpha+\beta(H / \sigma)$ through the least-squares fitting, where $\alpha$ and $\beta$ are the parameters. The ferrimagntic moment at $6 \mathrm{~K}, n_{\mathrm{f}}$, which is estimated from the square root of $\alpha$,

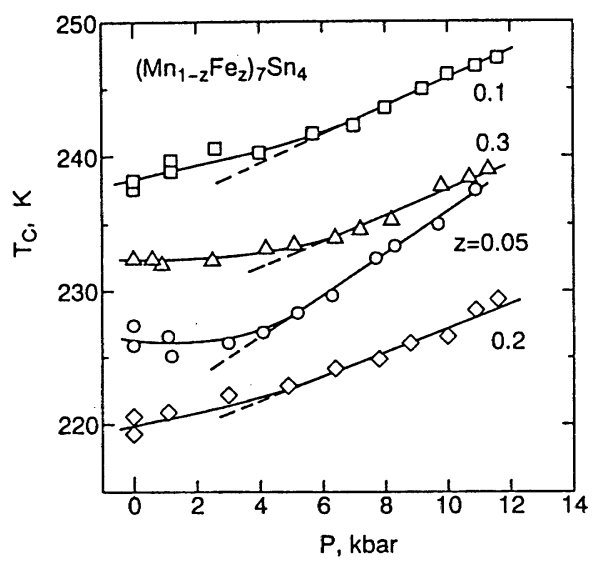

Fig.4. Curie temperature $T_{\mathrm{c}}$ as a function of pressure $P$ for various $z$ samples in $\left(\mathrm{Mn}_{1-2} \mathrm{Fe}_{2}\right)_{7} \mathrm{Sn}_{4}$.

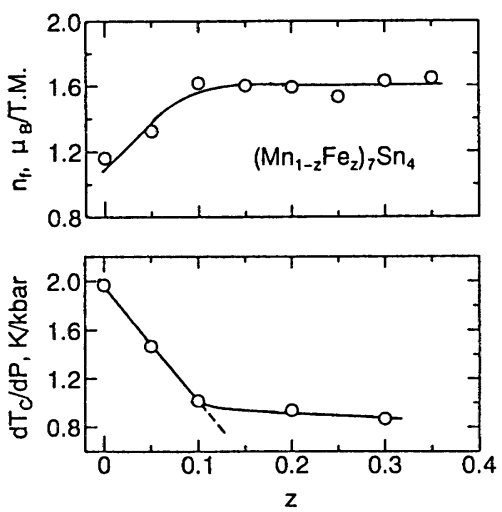

Fig.5. Ferrimagnetic moments at $6 \mathrm{~K}, n_{\mathrm{f}}$ (upper panel) and the pressure derivative of Curie temperature $T_{\mathrm{c}}, \mathrm{d} T_{\mathrm{c}} / \mathrm{d} P$ (lower panel), as a function of $z$ for $\left(\mathrm{Mn}_{1-2} \mathrm{Fe}_{2}\right)_{7} \mathrm{Sn}_{4}$.

initially increases up to about $z=0.1$ above which it hardly depends on $z$ in the range $0.1<z \leqq 0.35$ (the upper panel of Fig.5). As seen in the inset of Fig.6, $\beta$ for $\left(\mathrm{Mn}_{1-2} \mathrm{Fe}_{2}\right)_{7} \mathrm{Sn}_{4}$ and $\left(\mathrm{Mn}_{1-\mathrm{u}} \mathrm{Co}_{\mathrm{u}}\right)_{7} \mathrm{Sn}_{4}$ increase in a similar fashion while that for $\mathrm{Mn}_{7}\left(\mathrm{Sn}_{1-x} \mathrm{Ge}_{x}\right)_{4}$ remains constant.

\section{Discussion}

The values of lattice parameters $a$ and $c$ at room temperature linearly decrease with increasing $z$ (the lower panel of Fig.1), $u[7]$ and $x[11]$. 
The value of $n_{f}$ only slightly depends on $x$ but it steeply decreases with increasing $u[7,11]$. Then, it seems that $n_{\mathrm{f}}$ is mainly deternined with the number of $3 d$ electrons of metal atoms in the band features. This is because the total valence electrons does not vary with the change in $x[10]$. The number of $3 d$ electrons of $\mathrm{Fe}$ is intermediate between those of $\mathrm{Mn}$ and $\mathrm{Co}$. However, it is remarkbale that $\mathrm{d} n_{\mathrm{f}} / \mathrm{d} u<0$ while $\mathrm{d} n_{\mathrm{f}} / \mathrm{d} z>0(0 \leqq z \leqq$ $0.1)$. Hence, these features of $T_{\mathrm{c}}$ and $\eta_{\mathrm{f}}$ would be related to the difference in electron correlation $(I)$ nature of $3 d$ electrons in $\mathrm{Fe}$ and $\mathrm{Co}$ impurities. For $\mathrm{Mn}_{7}\left(\mathrm{Sn}_{1 \cdot x} \mathrm{Ge}_{x}\right)_{4}[10]$ and $\left(\mathrm{Mn}_{1}\right.$. $\left.{ }_{u} \mathrm{Co}_{u}\right)_{7} \mathrm{Sn}_{4}[8]$, the values of $\mathrm{d} T_{\mathrm{c}} / \mathrm{d} P$ are fairly described with a

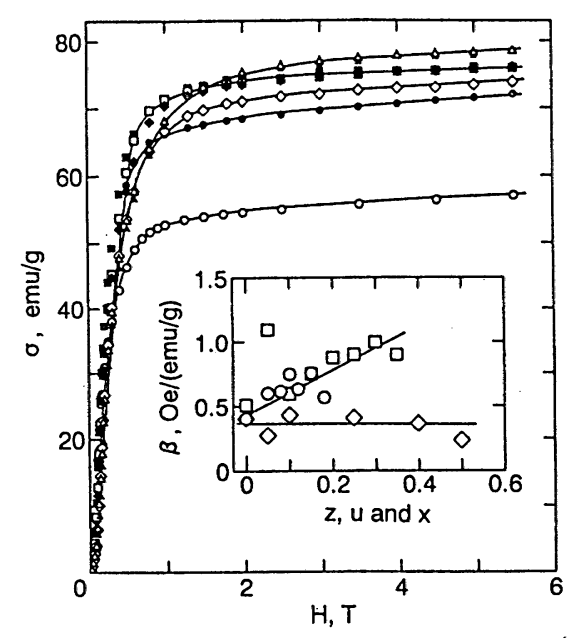

Fig.6. Magnetization $\sigma$ as a function of magnetic field $H$ at $6 \mathrm{~K}$ for various $z$ samples in $\left(\mathrm{Mn}_{1-2} \mathrm{Fe}_{2}\right)_{7} \mathrm{Sn}_{4}, \mathrm{O}: z=0$; 0.05 ;

$: 0.1 ; \square: 0.15 ; \diamond: 0.2 ; \diamond: 0.25 ; \Delta: 0.3 ; \triangle: 0.35$. Inset shows the Arrott plot's parameter $\beta$ (see text) for $\left(\mathrm{Mn}_{1-2} \mathrm{Fe}_{7}\right)_{7} \mathrm{Sn}_{4}(\square)$, $\left(\mathrm{Mn}_{1-u} \mathrm{Co}_{u}\right)_{7} \mathrm{Sn}_{4}(\mathrm{O})$ and $\mathrm{Mn}_{7}\left(\mathrm{Sn}_{1-x} \mathrm{Ge}_{x}\right)_{4}(\diamond)$.

relation[12] concerning electron correlation in itinerant electron ferromagnetic systems:

$\frac{\mathrm{d} T_{\mathrm{c}}}{\mathrm{d} P}=\mathrm{A} T_{\mathrm{c}}-\frac{\mathrm{B}}{T_{\mathrm{c}}}$.

Here, $A$ and $B$ are the parameters. In eq.(1), the first term is dominant in the case of strongly correlated electron ferromagnetic system, while the second term is dominant in weakly correlated one. As seen on the $T_{\mathrm{c}}\left(\mathrm{d} T_{\mathrm{c}} / \mathrm{d} P\right)$ vs $T_{\mathrm{c}}{ }^{2}$ curves(Fig.7), the values observed in $\left(\mathrm{Mn}_{1-2} \mathrm{Fe}_{2}\right)_{7} \mathrm{Sn}_{4}$ populate in the significantly lower region from the curves for $\left(\mathrm{Mn}_{1}\right.$ $\left.{ }_{u} \mathrm{Co}_{u}\right)_{7} \mathrm{Sn}_{4}$ and $\mathrm{Mn}_{7}\left(\mathrm{Sn}_{1-x} \mathrm{Ge}_{x}\right)_{4}$. The upper panel of Figure 1 also shows $\mathrm{d} T_{\mathrm{c}} / \mathrm{d} z>0$ above $z \sim 0.25 ; T_{\mathrm{c}}(z=1)$ is estimated to be $7 \times 10^{2} \mathrm{~K}$ by extrapolating to $\mathrm{z}=1$. Moreover, the value of $n_{\mathrm{f}}\left(=1.6 \mu_{\mathrm{B}}\right)$ in the range $0.25<z<0.35$ is comparable with that $\left(1.8 \mu_{\mathrm{B}}\right)$ in $\mathrm{Fe}_{1+\delta} \mathrm{Sn}[5]$. Therefore, it is plausible that $\mathrm{Fe}$ impurities retain their local magnetic character having the stronger $I$ than that of Co impurities in $\mathrm{Mn}_{7} \mathrm{Sn}_{4}$. More microscopic studies such as NMR and Mössbauer effect are highly desired to discuss the concrete physical states of $\mathrm{Fe}$ impurities.

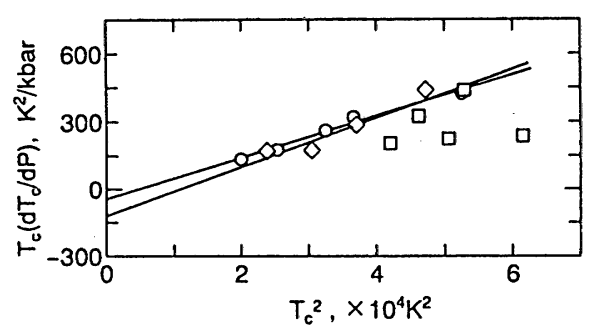

Fig.7. $T_{\mathrm{c}}\left(\mathrm{d} T_{\mathrm{c}} / \mathrm{d} P\right)$ against $T_{\mathrm{c}}{ }^{2}$ plots for $\left(\mathrm{Mn}_{1-2} \mathrm{Fe}_{2}\right)_{7} \mathrm{Sn}_{4}(\square)$, $\left(\mathrm{Mn}_{1-u} \mathrm{Co}_{u}\right)_{7} \mathrm{Sn}_{4}(\mathrm{O})$ and $\mathrm{Mn}_{7}\left(\mathrm{Sn}_{1-x} \mathrm{Ge}_{x}\right)_{4}(\diamond)$.

According to the spin fluctuation theory[13] for itinerant ferromagnetism, the parameter $\beta$ gives the information on the density of states at the Fermi level $E_{\mathrm{F}}, D\left(E_{\mathrm{F}}\right)$, and its energy derivative. Therefore, it is also noted that $\mathrm{d} \beta / \mathrm{d} z \fallingdotseq \mathrm{d} \beta / \mathrm{d} u$ besides $d n_{\mathrm{f}} / \mathrm{d} z>0$ and $\mathrm{d} n_{\mathrm{f}} / \mathrm{d} u<0$, although the theoretical relation bewteen

$\beta$ and $D\left(E_{\mathrm{F}}\right)$ is not known on the itinerant antiferomagnetic system.

Part of this work was carried out under the Visiting Researcher's Program of the Institute for Materials Research, Tohoku University.

\section{References}

[1] K.Yasukochi, K.Kanematu and T.Ohoyama, J.Phys.Soc. Jpn., 16,1123(1961).

[2] N.S.Satya-Murthy, R. J.Begun, B.S.Srinivasan and M.R.L. N.Murthy, Phys.Lett., 15, 225(1965).

[3] R.Sugi, T.Shimomura, M.Matoba, S.Matuzaka, S.Fujii, N.Hagiwara and S.Anzai, Phys.Stat.Sol., b 189,K65(1995).

[4] K.Ozawa, M.Ogawa, S.Yanagisawa and S.Anzai, Phys.Stat. Sol., 42, 787(1970).

[5] M.Asanuma, J.Phys.Soc.Jpn., 15,1343(1960).

· [6] H.Yamamoto, J.Phys.Soc.Jpn., 21 ,1058(1966).

[7] N.Tokiwa, T.Yoshida, T.Shimomura, R.Sugi, M.Matoba and S.Anzai, Phys.Stat.Sol., b189,K33(1995).

[8] T.Yoshida, H.Yoshida, R.Sugi, Y.Isogai, N.Tokiwa, S.Anzai, M.Matoba, T.Kaneko, S.Abe and S.Ohta, J.Phys. Soc.Jpn., 65,1511(1996).

[9] N.Hagiwara, M.Matoba, S.Fujii and S.Anzai, Phys.Stat. Sol. b 176, K 71(1993).

[10] S.Ohta, N.Hagiwara, S.Fujii, H.Yoshida, T.Kaneko, S.Anzai and M.Matoba, Jpn.J.Appl.Phys.,32,Suppl.32-3, 266 (1993).

[11] S.Anzai, N.Hagiwara, S.Fujii, S.Kameyama, M.Matoba, T.Kaneko , H.Yoshida and S.Ohta, Phys.Stat.Sol. b 177, K13 (1993).

[12] L.R.Edwards and L.C.Bartel, Phys.Rev., B 5, 1064(1972).

[13] T.Moriya, Spin Fluctuations in Itinerant Electron Magnetism, Springer, Berlin, 1985, p.77. 